\title{
Ontology-Based Inference for Supporting Clinical Decisions in Mental Health
}

\author{
Diego Bettiol Yamada ${ }^{1(\bowtie)}$, Filipe Andrade Bernardi ${ }^{2}$, \\ Newton Shydeo Brandão Miyoshi ${ }^{1}$, Inácia Bezerra de Lima ${ }^{3}$, \\ André Luiz Teixeira Vinci ${ }^{1}$, Vinicius Tohoru Yoshiura ${ }^{1}$, \\ and Domingos Alves ${ }^{1}$ \\ ${ }^{1}$ Ribeirao Preto Medical School, University of Sao Paulo, Ribeirao Preto, Brazil \\ diego.yamada@usp.br \\ ${ }^{2}$ Bioengineering Postgraduate Program, University of Sao Paulo, \\ Sao Carlos, Brazil \\ ${ }^{3}$ School of Nursing of Ribeirão Preto, University of Sao Paulo, \\ Ribeirao Preto, Brazil
}

\begin{abstract}
According to the World Health Organization (WHO), mental and behavioral disorders are increasingly common and currently affect on average $1 / 4$ of the world's population at some point in their lives, economically impacting communities and generating a high social cost that involves human and technological resources. Among these problems, in Brazil, the lack of a transparent, formal and standardized mental health information model stands out, thus hindering the generation of knowledge, which directly influences the quality of the mental healthcare services provided to the population. Therefore, in this paper, we propose a computational ontology to serve as a common knowledge base among those involved in this domain, to make inferences about treatments, symptoms, diagnosis and prevention methods, helping health professionals in clinical decisions. To do this, we initially carried out a literature review involving scientific papers and the most current WHO guidelines on mental health, later we transferred this knowledge to a formal computational model, building the proposed ontology. Also, the Hermit Reasoner inference engine was used to deduce facts and legitimize the consistency of the logic rules assigned to the model. Hence, it was possible to develop a semantic computational artifact for storage and generate knowledge to assist mental health professionals in clinical decisions.
\end{abstract}

Keywords: Ontology-based inference $\cdot$ Knowledge representation $\cdot$ Mental health

\section{Introduction}

Mental and behavioral disorders represent a large part of public health problems worldwide, it is estimated that by 2030 depression will be the largest cause of disability on the planet [1]. In countries such as the United States and Canada, it is estimated that this type of disorder is already the leading cause of disability for people aged 15-44 [2]. In Brazil, the situation is even more complicated, as the country lacks a formal and 
standardized model of mental health information, which makes it difficult for health professionals involved in this area to generate and use knowledge [3]. In Brazil, the Unified Health System ('SUS'; Portuguese: 'Sistema Único de Saúde') offers integral treatment at all levels of care, and these treatments often involve multi-professional care and procedures performed in different health services. Thus, it is essential to have a standardized model that facilitates communication between the various nodes of the health network, to help the units to communicate efficiently [4].

In this scenario, interoperability between Health Information Systems (HIS) presents itself as a viable solution to promote information sharing, exchange, and reuse [5]. Interoperability can be classified into four levels: fundamental, structural, semantic and organizational. Fundamental interoperability occurs through the exchange of data between HIS without the receiver having the ability to interpret the data. Its main applications range from direct database connections and service-oriented architectures using, for example, web services. Structural interoperability is an intermediate level that defines the syntax of data exchange to ensure that data exchanged between HIS can be interpreted and its meaning preserved and unchanged. Its main feature is based on the concept of corporate service buses using standards for message formats, for example, Digital Imaging and Communications in Medicine (DICOM) and Health Level Seven (HL7) [6].

The highest level of interoperability is achieved by the semantic level when two or more systems or elements can exchange information [7]. To achieve this, initially it is necessary to establish a standard structure of vocabularies and terminologies, expressive relations and description of processes for knowledge representation. One way to provide this type of entity standardization and taxonomy for HIS integration is by building and implementing computational ontologies [8]. By definition, an ontology is a set of explicit formal specifications of terminologies (classes or entities) and relationships (properties) between these elements in a given domain of knowledge [9]. Ontologies have contributed to facilitate the processing of information with added semantic value, mediating the exchange of information between machines and humans through computer systems and serving as a schema for knowledge bases, smart applications, health observatories, and inference knowledge models [7, 8].

Finally, we can also consider organizational interoperability, which is concerned with how different organizations collaborate to achieve their objectives by maintaining different internal structures and varying business processes. Even with the standardization of concepts and terminologies, organizations have different operating models or work processes. Thus, standardization of work processes, where two organizations need to have the same vision, is called organizational interoperability [3, 7].

In addition to the interoperability problems involving the mental health domain, it has been found that clinical decisions are often made without consulting a consolidated knowledge base [4, 10]. Therefore, semantic applications, such as ontologies, are relevant in health because besides having originally integrated knowledge, this tool can be constantly developed with the dynamic participation of the community that involves this domain of knowledge.

Decision support tools are essential to guide the practice of health care and support the decisions that will directly influence the quality of care provided to the population. Making a reference data set available as a Decision Support System (DSS), provided 
with consistent ontologies, for integrating, analyzing, comparing and viewing health data through the integration of heterogeneous and dispersed databases is the main aspect to be considered [11].

In this way, we merge the best practices of ontology development with the knowledge collected on mental health to build an expressive semantic repository through upper-ontology [8, 12]. The World Wide Web Consortium (W3C) best practices for building semantic applications involve 3 basic blocks: a standard data model; a query protocol; and a set of reference vocabulary and terminologies. Resource Description Framework (RDF), Simple Protocol and RDF Query Language (SPARQL), and ontologies developed in Ontology Web Language (OWL) refer to these basic blocks respectively [13]. Therefore, we consider that mental health processes will be more efficient if based on a formal structure that will be proposed through the use of the mentioned set of standards and technologies.

The main purpose of this paper is to develop a computational ontology capable of representing the reality of the mental health domain and making evidence-based inferences, assisting health professionals in clinical decisions and promoting semantic interoperability between mental health information systems. The rest of the paper is organized as follows. In Sect. 2, related studies on the use of ontologies to support clinical decisions will be discussed. In Sect. 3, the proposed methodology will be presented in detail. Experimental results will be presented in Sect. 4. Finally, the conclusion will be described in Sect. 5 .

\section{Literature Review}

The $\mathrm{W} 3 \mathrm{C}$ has been committed to projects to improve integration, standardization, and data sharing in the World Wide Web. Such initiatives involve the use of the Semantic Web, defined as a set of technologies that, not only links documents, but is also recognize the meaning of the data from these documents and, through ontologies, promote inferences that help management and decision making. Thus, ontologies have been used on a large scale in the medical field, as this domain presents a complex and dispersed data in HIS with low standardization and integration [5].

Many studies have identified that human errors are frequent in clinical settings. The three most common types of errors in these environments relate to non-compliance with guidelines, hasty decision-making, and lack of awareness of the responsibilities and roles of each type of practitioner [14]. These are problems that can easily be avoided by an ontology-based decision support system [15]. Cases of development of this type of application can be observed in various areas of health.

In midwifery, for example, the detection of characteristics that frame women in a risky pregnancy is essential for proper and personalized medical follow-up. However, in many countries, the lack of human resources in the medical field means that these diagnoses are performed without proper scrutiny, leading many women to death due to complications in pregnancy. Thus, in Pakistan, an ontology-based clinical decision support system has been developed to assist in the diagnosis of high-risk pregnant women and refer them to qualified physicians for timely treatment [15]. 
In the field of Alzheimer's disease, the early detection of pathology is a complex task for medical staff, usually, the approach involves medical image processing, psychological testing, and neurological testing, and such exams produce data that can generate knowledge. In the United States, one of the ways found to mitigate this complexity was by developing a tool to support clinical decision making, where ontologies and semantic reasoning play a key role in making inferences about diagnoses through the collected patient data [16].

In recent years the population has grown older and the number of patients with chronic respiratory-related diseases has increased [17]. In Taiwan, an intelligent ontology-based tool has been built to assist medical staff in recognizing changes in clinical examinations for the detection of chronic respiratory diseases. This system recognizes patterns that usually lead patients to develop respiratory problems and points out these characteristics to the medical team, who in possession of this information can more accurately indicate the degree of complexity of pathologies related to a patient's respiratory system [18].

Breast cancer is the most common cancer among women in many countries. In Canada, a Semantic Web-based application has been implemented to support physicians in tracking patients with this disease. The approach involves computerization and implementation of guidelines for monitoring women undergoing breast cancer treatment. For this, a domain ontology was built that models the knowledge inherent in these guidelines and practices and serves as a source of knowledge to determine specific recommendations for each patient [19].

Also, many other semantic applications already exist in healthcare and many more are under construction at this time. The justification for the development of these applications is the accessible implementation of these technologies in new and legacy systems, the low cost, and the encouragement of the use by various international entities to disseminate, integrate and reuse the knowledge generated [8, 15]. For the efficient use of a semantic tool, the information inserted in it must come from concrete and reliable sources. In this paper, we use studies and knowledge from the WHO's Mental Health Action Plan 2013-2020 [20] and the Diagnostic and Statistical Manual of Mental Disorders (DSM-5) [21].

\section{Methods}

In this paper, we propose a framework for inferring mental health knowledge through the use of a domain ontology. This framework consists of three main modules: 1) Structural Ontology Model, 2) Fact Inference Engine and 3) RDF Database.

\subsection{Structural Ontology Model}

An ontology is an explicit and formal specification of a set of concepts in a specific field of interest [9]. In this proposal, an ontology for the mental health domain was developed to assist in the management of the mental health care network in Brazil, to standardize concepts and to assist the integration and interoperability between different 
health units. Besides, this tool can infer knowledge from the inputted data in ontology to assist mental health professionals in clinical decisions.

The building of an ontology usually comprises a series of stages, therefore, for proper coordination of all these steps, the Methontology methodology was adopted. Developed at the Artificial Intelligence Department of the Polytechnic of Madrid (http://www.dia.fi.upm.es/), this ontology construction methodology stands out for presenting a rigid detail to explore and structure the processes of knowledge acquisition [22]. The knowledge acquisition phase for this project involved interviews with mental health professionals and a literature review that considered scientific articles, official documents, WHO guidelines and the DSM-5 standards and classifications.

Subsequently, we used the Protégé software (https://protege.stanford.edu/) to transfer the collected knowledge to the computational environment, thus building the desired ontology, with all its entities and properties developed in OWL, following the data model RDF. Protégé software has been selected for being open source, reliable and robust, and has wide acceptance and compatibility with other international projects [23].

Among the created entities, we store information about the types of mental disorders, symptoms, prevention methods, types of medications, side effects of these drugs, types of health units, management processes of psychiatric hospitals, documents that involve these processes, among other information from the knowledge acquisition phase. Information on mental disorder classes as well as their subtypes was imported from the 10th revision of the International Classification of Diseases (ICD-10) [24].

We then use the Jena Ontology Application Programming Interface (API) (https:// jena.apache.org/documentation/ontology/) to perform fact deduction and legitimation of the consistency of the logical rules assigned to the semantic repository. This programming toolkit uses the Java language to provide the developer with standardized structures that connect the modules of a semantic platform [25], as detailed in Sect. 3.2.

\subsection{Fact Inference Engine}

After the ontology was built, we uploaded the proposed schema on the web via the BioPortal platform (https://bioportal.bioontology.org/) and we imported this model into the Jena Fuseki Server (https://jena.apache.org/). Using the API provided by this software and the Hermit Reasoner inference engine (http://www.hermit-reasoner.com/) it was possible to validate the entities that make up the ontology, as well as all its connective elements, aiming to generate consistent facts from the information inserted in the ontology [26]. Figure 1 shows the scheme used in this step, arrows mean the connected modules consume information from each other.

The elements of ontology had their structure verified by the aforementioned inference engine, this step avoids future data inconsistency problems. Posteriorly, the information was entered into the RDF triples that make up the structure of the ontology. The next section details the operation of this type of data repository. 


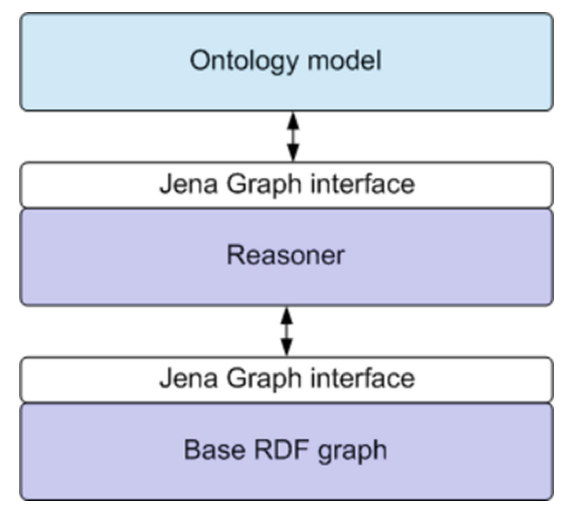

Fig. 1. Ontology-based application structure

\subsection{RDF Database}

Through Protégé software, we insert mental health information into the semantic repository schema created, then use the Jena Fuseki Server to create an endpoint to perform custom queries to the database using the SPARQL language. This query language has a syntax that encompasses commands capable of providing broad effectiveness in extracting content from a semantic base. Also, the results can be presented in various ways, according to the user's needs. Figure 2 shows how the search for information stored in triple RDF occurs.

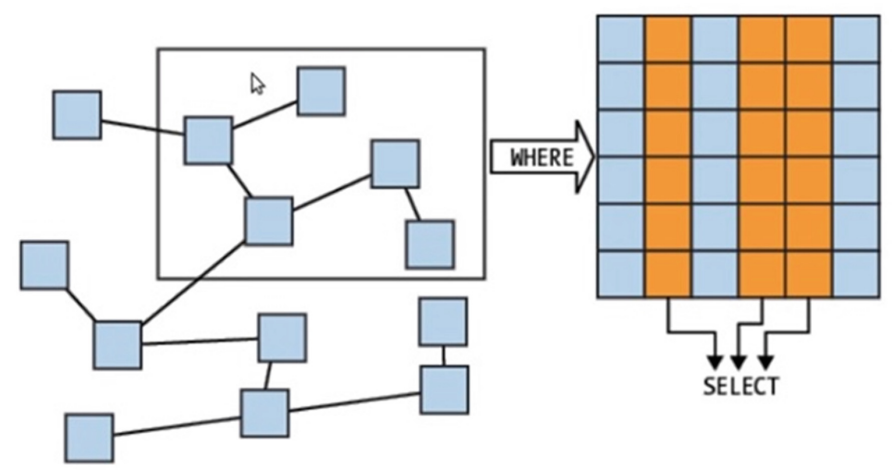

Fig. 2. Operation of a SPARQL query

The SELECT command is responsible for specifying the conditions for selecting the data relation under which the query will be executed, and the WHERE clause is responsible for selecting which data of this relation to display. This basic structure added to a range of other expressive commands allows the elucidation of facts and the asking of questions about knowledge stored in the triple form [27]. 
Thus, queries were held to test the use of the structure to prove its functionality to clarify relevant facts about the context of mental health. According to WHO, the use of contextual information about types of disorders, symptoms, treatments, preventions and drug side effects are essential topics for the continuous improvement of health services $[1,20]$. The results of this process are in the later section.

\section{Results and Discussion}

A series of tests were conducted to demonstrate the effective functioning of the proposal. In this section, we will present the main results of the work.

The experiments focused on elucidating facts about mental disorders and their symptoms, treatments, prevention recommendations and medications. According to WHO, this information is of great importance for clinical decisions within the mental health field [20]. To exemplify these features, in this paper, we chose depression as the target disorder. This disorder was chosen due to its high tendency of social and economic impact [1]. Besides, according to the Mental Health Information System ('SISAM'; Portuguese: 'Sistema de Informação de Saúde Mental'), which coordinates mental health in 26 Brazilian municipalities, mood disorders are the second leading cause of psychiatric hospitalizations in the region, accounting for $31 \%$ of all cases of psychiatric hospitalizations [3]. In the United States, depression is also one of the most common psychiatric disorders, with an estimated lifetime prevalence rate of $16.2 \%$ [28]. Figure 3 shows the location of the concept of "Mental Depression" in the structure of the ontology.

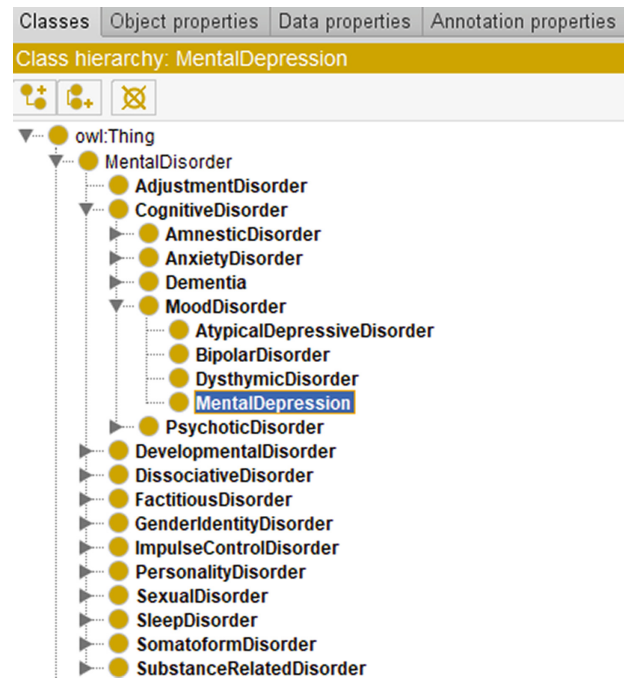

Fig. 3. Mental depression entity 
Therefore, we relate this disorder with the knowledge about it collected. Through the Property Assertions function of Protégé software, we use the relationships created to bind the classes with their respective instances, so finally we could perform SPARQL queries to extract knowledge.

Figure 4 shows how semantic inference is made through a SPARQL query that looks for the main symptoms of depression, using a standardized terminology according to the guidelines of WHO and DSM-5. We can see that the set of results provides relevant and helpful information for clinical decision making, as the structured knowledge of the most frequent symptoms presented by depressive patients provides the psychologist or psychiatrist with a consistent basis for better identification of the disorder [29].

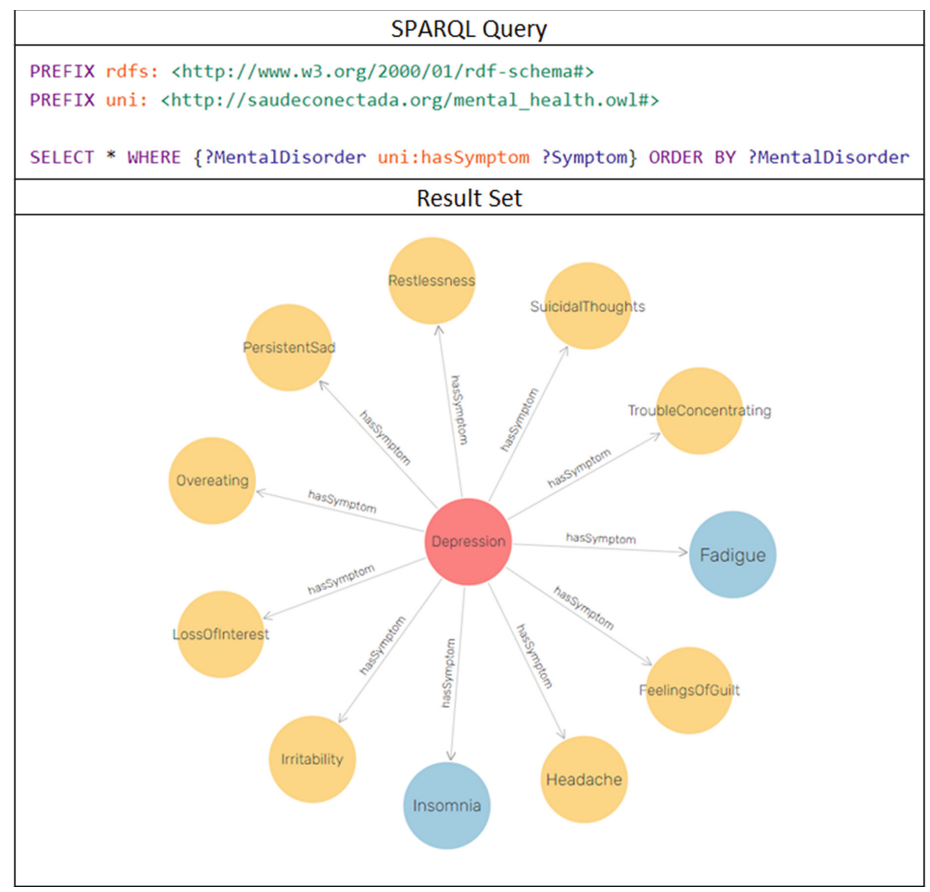

Fig. 4. Inference about depression symptoms

To diagnose depression, 5 of these symptoms should be present almost every day for 2 weeks, and one of them must be persistent sadness or loss of interest [21]. Therefore, the knowledge structured in the ontology is characterized as a solid repository of evidence-based information and supports the fast and accurate diagnosis identification, so the patient can then be referred to the most appropriate treatment.

Knowledge about the most recommended types of treatments for a disorder is also of great importance to aid a medical decision [20]. The entities referring to the different types of treatments for mental disorders are also structured in our ontology. Figure 5 
shows some classes of treatments associated with depression [21]. The standardization of this type of information avoids inconsistencies in data sharing between different health services $[11,15]$.

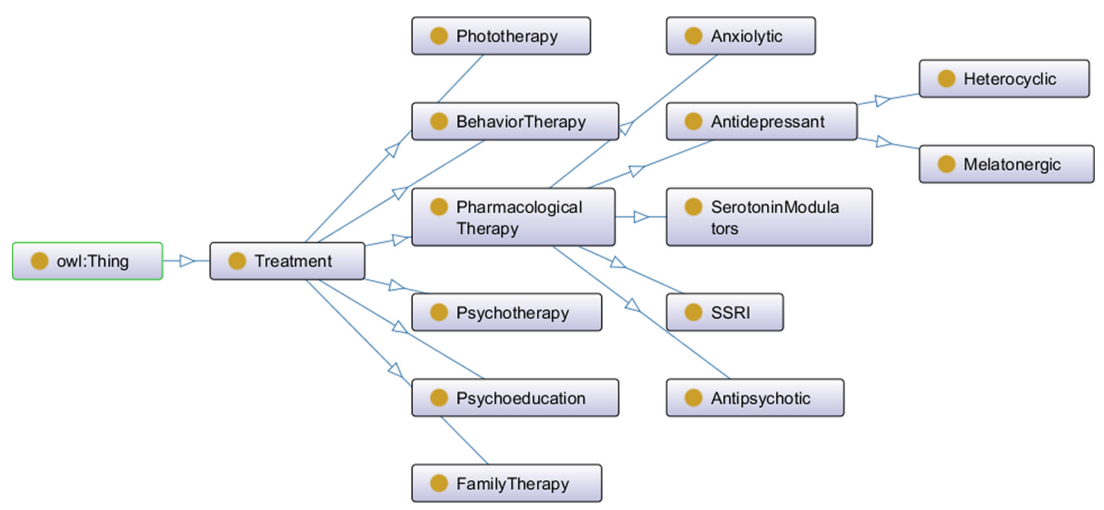

Fig. 5. Structure of treatments for depression

Each type of treatment has its characteristics, but knowledge about the consequences of recommending psychoactive drugs should also be highlighted in the model. Since on average $38 \%$ of patients who have already taken antidepressants have experienced at least one of the side effects of this drug [30] and these effects are more detrimental to long-term health [29], the healthcare professional must have at its disposal a standardized digital base with the possible side effects of each class of medicine. Figure 6 shows an inference about the main side effects related to the use of antidepressant drugs.

Other side effects, treatments, symptoms, drugs, disorders, or any entities may be inserted or removed from the platform as discoveries are made through scientific research, it is important to remember that this type of tool is available on the web for future updates and maintenance as needed. An ontology must represent the reality of a domain, whereas reality is changeable the ontology must also provide scope for alteration to correctly represent the desired domain [7, 9].

Considering the many side effects caused by the continued use of psychoactive drugs [29], and the currently increasing demand for mental health treatments and services [4], the public health policies are essential and must be focused on preventing these disorders, minimizing social problems and resource expenditures from more complex health services [30]. Therefore, our model also can infer prevention recommendations for mental disorders. Figure 7 shows the results of this type of query for depression.

It is important to emphasize that an ontology must minimize any kind of ambiguity or subjectivity in the representation of knowledge $[6,12]$. This way, many additional resources can be used to complement information stored in this type of tool. We can use, for example, semantic annotations of the RDF-Schema (RDF-S) data model, an extension of the RDF, to relate concepts of our vocabulary with other relevant information on the web. 


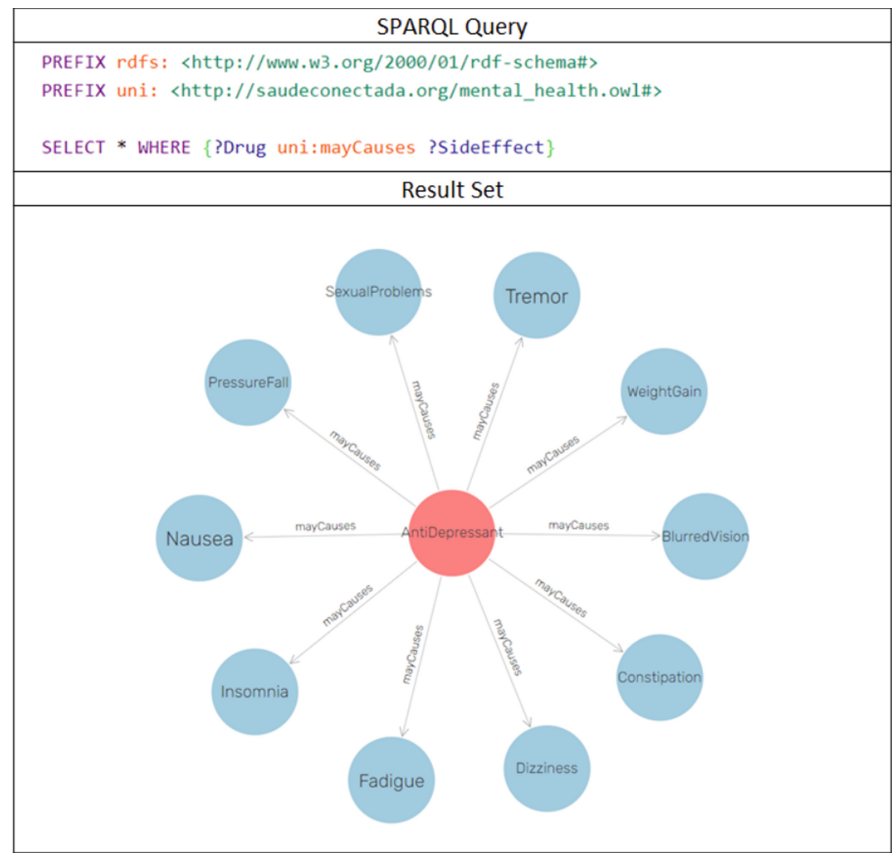

Fig. 6. Inference about antidepressant side effects

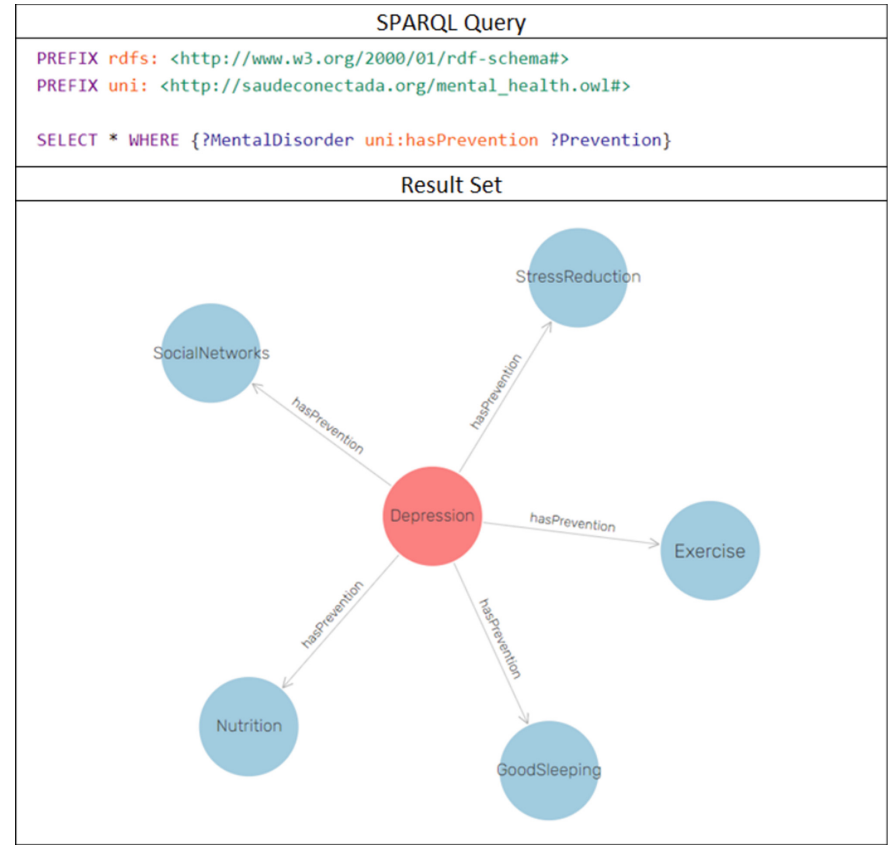

Fig. 7. Inference about prevention recommendations 
For example, the "GoodSleeping" concept, presented as a preventive aspect in the Result set of Fig. 7, can bring subjectivity about how much sleep would be ideal for each individual. Therefore, we can create semantic annotations for this concept, such annotations may refer to concepts from other web pages or other ontologies available on the web [9]. In this case, we use the National Sleep Foundation website (https:// www.sleepfoundation.org/) [31] to couple with this concept, thus highlighting the recommendations of sleep hours for each age group.

In this paper, specifically, we show results of depression-related inferences, but many other mental disorders can be explored. There are no limits to the knowledge stored in this platform, the semantic repository is dynamic and is available on the Web to be constantly fed with new knowledge from professionals and researchers involved in this area.

The developed ontology comprises 361 classes, 37 relationships among these classes, 72 individuals, and the maximum depth is 9 . This proposal not only addresses the biomedical aspects of mental health but also relates them to the day-to-day operations of health services, allowing information systems to be aligned with the strategies of the mental health network, which is its most innovative point. Even in a clinical setting supported by the use of traditional information systems and electronic medical records, the rate of medical errors can still reach $24.4 \%$ [32]. In this way, computational ontologies are presented as an extension of these traditional systems, offering additional ways to structure information, infer and make available evidence-based knowledge.

However, ontology still needs the insertion of a wider range of knowledge and the establishment of relationships with other health areas, thus increasing its completeness. For example, drug entities may be related to other pharmaceutical ontologies, providing the exact description of each type of medication and their characteristics. Nutritionrelated entities can be connected with other nutritionism ontologies, thus providing highly expressive and informative structures to users.

Aiming at this kind of integration, we make available the developed ontology (Mental Health Management Ontology - MHMO) in the international repository of BioPortal biomedical ontologies so that it can be used in other health projects around the world. The BioPortal is an open database that provides access to biomedical ontologies via Web services, facilitating community participation in ontology assessment and evolution and providing additional resources for terminology mapping and criteria review [33].

\section{Conclusion}

In conclusion, this computational artifact contains stored knowledge that can be extracted through personalized consultations to assist mental health professionals in clinical decisions and can be used as a common basis for knowledge sharing between humans and machines, promoting improvements in interoperability issues of HIS. It can also be used as a layer for building other decision-support information systems, health observatories, and varied smart applications, being useful in areas such as machine learning and artificial intelligence. 
This study is part of a larger project. As future work, we aim to use the ontology to develop a Mental Health Observatory for a Brazilian Public Health Network, to improve the monitoring, analysis, and visualization of mental health issues, providing information to support evidence-based decisions, health policies elaboration, public planning, and data sharing. The ontology can be constantly fed with new knowledge to continuously assist the mental health services provided to the population and can be used to optimizing processes, reducing resource consumption and bringing benefits to health professionals, managers, and patients.

\section{References}

1. World Health Organization - The global burden of disease. http://www.who.int/entity/ healthinfo/global_burden_disease/GBD_report_2004update_full.pdf. Accessed 04 Dec 2019

2. National Advisory Mental Health Council Workgroup - From discovery to cure: Accelerating the development of new and personalized interventions for mental illness. https://www.nimh.nih.gov/about/advisory-boards-and-groups/namhc/reports/ fromdiscoverytocure_103739.pdf. Accessed 04 Dec 2019

3. Miyoshi, N.S.B., De Azevedo-Marques, J.M., Alves, D., De Azevedo-Marques, P.M.: An eHealth platform for the support of a Brazilian regional network of mental health care (eHealth-Interop): development of an interoperability platform for mental care integration. JMIR Ment. Health 5(4), e10129 (2018)

4. Castro, S.A.D.: Adesão ao tratamento psiquiátrico, após alta hospitalar: acompanhamento na rede de serviços de saúde (Doctoral dissertation, Universidade de São Paulo) (2015)

5. Yoshiura, V.T., et al.: Towards a health observatory conceptual model based on the semantic web. Proc. Comput. Sci. 138, 131-136 (2018)

6. Jabbar, S., Ullah, F., Khalid, S., Khan, M., Han, K.: Semantic interoperability in heterogeneous IoT infrastructure for healthcare. Wireless Commun. Mob. Comput. 2017 (2017)

7. Abhishek, K., Singh, M.P.: An ontology based decision support for tuberculosis management and control in India. Int. J. Eng. Technol. 8(6), 2860-2877 (2016)

8. Yamada, D.B., et al.: Proposal of an ontology for mental health management in Brazil. Proc. Comput. Sci. 138, 137-142 (2018)

9. Shaaban, A.M., Gruber, T., Schmittner, C.: Ontology-based security tool for critical cyberphysical systems. In: Proceedings of the 23rd International Systems and Software Product Line Conference, vol. B, p. 91. ACM, September 2019

10. Vinci, A.L.T., Rijo, R.P.C.L., de Azevedo Marques, J.M., Alves, D.: Proposal of an evaluation model for mental health care networks using information technologies for its management. Proc. Comput. Sci. 100, 826-831 (2016)

11. Delfini, M. ., Miyoshi, N.S.B., Alves, D.: Minimum data consensus: essential information to continuing healthcare. In: 2015 IEEE 28th International Symposium on Computer-Based Medical Systems, pp. 205-207. IEEE, June 2015

12. Willner, A., et al.: The open-multinet upper ontology towards the semantic-based management of federated infrastructures. EAI Endor. Trans. Scalable Inf. Syst. 2(7) (2015)

13. W3C Semantic Web Standards. https://www.w3.org/standards/semanticweb/. Accessed 11 Dec 2019

14. Sanchez, E., et al.: A knowledge-based clinical decision support system for the diagnosis of Alzheimer disease. In: 2011 IEEE 13th International Conference on e-Health Networking, Applications and Services, pp. 351-357. IEEE, June 2011 
15. Manzoor, U., Usman, M., Balubaid, M.A., Mueen, A.: Ontology-based clinical decision support system for predicting high-risk pregnant woman. System 6(12), 203-208 (2015)

16. Adnan, M., Warren, J., Orr, M.: Ontology based semantic recommendations for discharge summary medication information for patients. In: 2010 IEEE 23rd International Symposium on Computer-Based Medical Systems (CBMS), pp. 456-461. IEEE, October 2010

17. Zwar, N., et al.: A systematic review of chronic disease management (2017)

18. Lee, C.-S., Wang, M.-H.: An ontology-based intelligent agent for respiratory waveform classification. In: Ali, M., Dapoigny, R. (eds.) IEA/AIE 2006. LNCS (LNAI), vol. 4031, pp. 1240-1248. Springer, Heidelberg (2006). https://doi.org/10.1007/11779568_131

19. Abidi, S.R., Hussain, S., Shepherd, M., Abidi, S.S.R.: Ontology-based modeling of clinical practice guidelines: a clinical decision support system for breast cancer follow-up interventions at primary care settings. In: Medinfo 2007: Proceedings of the 12th World Congress on Health (Medical) Informatics; Building Sustainable Health Systems, p. 845. IOS Press (2007)

20. World Health Organization: Mental health action plan 2013-2020 (2013)

21. American Psychiatric Association. Diagnostic and statistical manual of mental disorders (DSM-5). American Psychiatric Pub (2013)

22. González, M.A.V.: Building ontologies with methontology as a technical resource for specialized translation: ontoUAV project, a multilingual web ontology (EN/FR/ES) on Unmanned Aircraft Vehicles (UAV) for specialized translation. In: Temas actuales de terminología y estudios sobre el léxico, pp. 163-192. Comares (2017)

23. Musen, M.A.: The protégé project: a look back and a look forward. AI Matters 1(4), 4 (2015)

24. WHO ICD 10 online version. https://www.who.int/classifications/icd/icdonlineversions/en/. Accessed 11 Dec 2019

25. Selvaraj, S., Choi, E.: A study on traditional medicine ontology. In: Proceedings of the 2nd International Conference on Software Engineering and Information Management, pp. 235239. ACM, January 2019

26. Shearer, R., Motik, B., Horrocks, I.: HermiT: a highly-efficient OWL reasoner. In: Owled, vol. 432, p. 91 , October 2008

27. Allemang, D., Hendler, J.: Semantic Web for the Working Ontologist: Effective Modeling in RDFS and OWL. Elsevier, Amsterdam (2011)

28. Kessler, R.C., et al.: The epidemiology of major depressive disorder: results from the National Comorbidity Survey Replication (NCS-R). JAMA 289(23), 3095-3105 (2003)

29. Karyotaki, E., et al.: Combining pharmacotherapy and psychotherapy or monotherapy for major depression? A meta-analysis on the long-term effects. J. Affect. Disord. 194, 144-152 (2016)

30. Cascade, E., Kalali, A.H., Kennedy, S.H.: Real-world data on SSRI antidepressant side effects. Psychiatry (Edgmont) 6(2), 16 (2009)

31. National Sleep Foundation Recommends New Sleep Times. https://www.sleepfoundation. org/press-release/national-sleep-foundation-recommends-new-sleep-times. Accessed 12 Dec 2019

32. Shojania, K.G., Burton, E.C., McDonald, K.M., Goldman, L.: Changes in rates of autopsydetected diagnostic errors over time: a systematic review. JAMA 289(21), 2849-2856 (2003)

33. Salvadores, M., Alexander, P.R., Musen, M.A., Noy, N.F.: BioPortal as a dataset of linked biomedical ontologies and terminologies in RDF. Semant. Web 4(3), 277-284 (2013) 